Open space questions and word cloud responses also highlighted qualitative feedback with most frequent responses including 'inspiring', 'interesting and 'stimulating'.

174 posters were presented with good representation from all services and staff groups across the trust (18 on safer use of medicines, 15 on co-production, 52 on quality improvement, 50 on COVID and non-COVID safety, 16 on use of technology, 23 on supporting and involving staff). These posters have since been downloaded 4062 times.

Conclusion. The first CNWL safety conference proved an excellent opportunity to celebrate achievements in patient safety in a very difficult year. It was very well-received and well-attended by staff, promoting maximal learning across the organisation.

Whistle while you work: improving psychiatry training in a London NHS Trust and what we learned along the way

Emily Duncan*, Alex Bailey, Simon Edwards, Alison Butler and Layth Humsi

Central and Northwest London NHS Trust

${ }^{*}$ Corresponding author.

doi: 10.1192/bjo.2021.383

Aims. The aim of this project is to improve the training experience of Psychiatry trainees across CNWL. In QI terms, we want to achieve a satisfaction rating of above $7 / 10$ for all themes identified by August 2021.

Method. Collected baseline data on satisfaction and priority ratings on 7 training themes Held discussion groups with trainees for specific themes to generate issues and solutions Developed and provided Quality Improvement training for trainees and trainers, 1:1 support and QI clinics - empowering trainees to develop their own local project and to make changes to issues on the ground Enacted central changes in communication, responsiveness, recognising success.

Reassessed and fedback to the trainees throughout.

Result. Our baseline satisfaction survey was completed in June 2020. Trainees their satisfaction for each theme out of 10 and to rank their priorities for change. Results showed satisfaction was lowest in morale and in safety and highest in education and supervision. Their highest priorities for change were safety, then morale, with induction as the lowest priority.

We repeated the survey in October 2020. This showed improvements in most themes (apart from induction, perhaps due to induction having to be delivered virtually). Satisfaction in key priority areas of morale and safety increased from 4.53 to 6.37 , and 5.12 to 6.70 respectively. We also asked what 'one thing' would they improve about their training. Key phrases included teaching, on-call, communication and induction.

From this data, and softer feedback from trainees, it is encouraging that we are moving in a positive direction, but we are continuing to make changes.

Conclusion.

- Trainees must be central to the work in improving their training

- Using QI methodology helps - developing a structure and breaking down a bigger task helps make a plan

- Feedback is key - but people are busy and receive a lot of emails and requests to fill surveys - catching people 'in person' (virtually) was the best way to ensure a lot of responses

- Trainees have loads of great ideas, but they need support, time and resources to be able to develop their projects and changes
- Flexibility is crucial: some topics work better locally, driven by trainees and some require a more coordinated, central role

We hope that developing a structured approach to a large task like improving training will help make changes sustainable, and enables us to share our learning with others.

\section{Tackling constipation in patients on high dose antipsychotics}

Sandhya Eappen*

Parklands Hospital

${ }^{*}$ Corresponding author.

doi: 10.1192/bjo.2021.384

Aims. The aim of this audit was to look into patients on high dose antipsychotics who had developed complications of constipation in the PICU setting .

Background. Antipsychotics are usually used in the treatment of Schizophrenia and other psychotic illnesses. Drug such as Clozapine mainly has a higher risk profile due to gastrointestinal hypo motility. It could present as constipation, fecal impaction or a bowel obstruction and could even lead to death.

Method. During ward rounds enquired on bowel habits and diet.

Physical examination of patients complaining of abdominal pain.

Screened notes in past to see how many patients complained of constipation and interventions suggested and used.I65.

Result. 3 of the 10 patients on PICU were on high dose antipsychotics and 2 of them had reported constipation. Of which one required daily review and vigorous treatment with laxatives and dietary changes.

Recommendation

Bristol stool chart introduced as part of care plan for all patients.

Teaching presentation of constipation and its treatment management was given to the PICU team.

Involving medical team early on for assessment and prophylactic laxatives prescription.

Liaison with the pantry team to include more options of fruits and vegetables into daily meal plan for patients.

Data and material handed over to next trainee to Re-audit and complete audit cycle.

Conclusion. Appropriate prevention and early management of side effects can enhance the benefits of antipsychotics. Bowel function monitoring and the use of prophylactic laxatives for patient on high dose antipsychotics such as clozapine is advisable to prevent complications related to it.

\section{Bridging the gap - MTI experience to an excellent MTI experience - An experiential, theme driven, concordance verifying study}

Arun Enara ${ }^{1 \star}$, Kabir Garg ${ }^{2}$ and Ramachandran Kanchana ${ }^{1}$

${ }^{1}$ Hertfordshire partnership Foundation NHS trust and ${ }^{2}$ Oxleas NHS Foundation Trust

${ }^{*}$ Corresponding author.

doi: 10.1192/bjo.2021.385

Aims. To collate experiences of international medical graduates (trained psychiatrists) on the Medical Training Initiative (MTI) and equivalent programs (International Medical Fellowship 